\title{
Mechanisms of L-lysine extraction with sec-octylphenoxy acetic acid in sulfonated kerosene
}

\author{
Chunhua Zhao, ${ }^{a}$ Xiaohua Zhou, ${ }^{a *}$ Yan Xiao, ${ }^{b}$ Dan Wang, ${ }^{a}$ Zhen Zhou, ${ }^{c}$ \\ Zhixiong Yang, ${ }^{a}$ Xiaoqin Jiang ${ }^{a}$ and Jiang Wang ${ }^{a}$
}

\begin{abstract}
BACKGOUND: Currently, the dominant theories on extraction mechanisms of amino acids are mainly deduced from mathematical models or reaction equations, it is thus necessary to obtain direct evidence for the extraction mechanisms by experimental methods. This work aims to reveal the mechanisms of L-lysine (L-Lys) extraction with sec-octylphenoxy acetic acid (CA-12) in sulfonated kerosene by atomic force microscopy (AFM), half-saturated fluorometric experiment and competition experiment.

RESULTS: The ionic bond based on the electrostatic attraction between the amino group of L-Lys and the carboxyl group of CA-12 was verified visually by high-resolution AFM. Half of the amino groups of L-Lys were labeled by o-phthalaldehyde (OPA) to form half-saturated fluorescent derivatives which were then extracted by means of CA-12 in sulfonated kerosene. The electrostatic attraction between the free amino group of L-Lys and the carboxyl group of CA-12 was illustrated by the relationships between OPA dosage and fluorescence intensities of stock solution and extracted liquid. This electrostatic interaction was also demonstrated by the competition experiment using three other amino acids including L-arginine, L-aspartic acid and L-alanine as competitive reagents.
\end{abstract}

CONCLUSION: The chemical force of L-Lys extraction with CA-12 in sulfonated kerosene was comprehensively demonstrated to be the electrostatic interaction between the amino group and the carboxyl group.

(c) 2015 Society of Chemical Industry

Keywords: L-lysine; sec-octylphenoxy acetic acid; extraction mechanism; electrostatic interaction; AFM

\section{INTRODUCTION}

Liquid-liquid extraction (LLE) is a process of transferring a chemical compound from one liquid phase to the other liquid phase. ${ }^{1-3}$ In recent decades, LLE has been becoming more attractive for separation and purification of amino acids due to the high extraction efficiency, saving in solvent and energy, possibility of room temperature operation, continuous production and low cost. ${ }^{4-7}$ Amino acids dissociate in aqueous solutions, forming characteristic ionic species (i.e. the cation $\mathrm{A}^{+}$, zwitterion $\mathrm{A}^{ \pm}$and anion $\mathrm{A}^{-}$) as a function of the solution $\mathrm{pH}$ value. As a result, the solubility in nonpolar solvents is very low. Therefore, LLE of amino acids is generally achieved by adding the organic phase extractants such as phosphoric acid derivatives, ${ }^{8}$ quaternary aliphatic amines ${ }^{9}$ or crown ethers, ${ }^{10}$ etc. mainly including the method of ion-exchange reaction extraction, liquid membrane extraction or reversed micelle extraction.

There are four theories on the extraction mechanisms of amino acids including electrostatic interaction, ${ }^{11-13}$ Lewis acid-base interaction, ${ }^{14-17}$ proton transfer reaction ${ }^{18-22}$ and complexation extraction. ${ }^{23-27}$ The electrostatic interaction was involved in many amino acids extraction either using acidic extractant (e.g. di(2-ethylhexyl) phosphoric acid (D2EHPA)) ${ }^{8}$ or basic extractant (e.g. trioctylamine (TOA)) ${ }^{14}$ or using reversed micelles formed by anionic surfactant (e.g. bis(2-ethylhexyl) sulfosuccinate sodium salt (AOT) $)^{28,29}$ or cationic surfactant (e.g. trioctylmethylammonium chloride $(\mathrm{TOMAC})^{9}$ ). The Lewis acid-base interaction was a more extensive theory which might refer to ion exchange and proton transfer in amino acids extraction using Lewis acid extractant (e.g. D2EHPA) or Lewis basic extractant (e.g. TOA). ${ }^{14,30}$ The proton transfer reaction theory mainly concerned the extraction and separation of electrically neutral amino acids (i.e. amino zwitterionic molecules). Liu etal. demonstrated that the proton-transfer reaction occurred in the extraction of $\alpha$-amino acid and explained the complicated phenomenon present in the extraction of L-tryptophane with D2EHPA dissolved in n-octane and n-octanol. ${ }^{18}$ They found Lewis acid D2EHPA in the organic phase

Correspondence to: Xiaohua Zhou, College of Chemistry and Chemical Engineering, Chongqing University, Chongqing 400044, China.

E-mail:zhou65306590@cqu.edu.cn

a College of Chemistry and Chemical Engineering, Chongqing University, Chongqing 400044, China

b Department of Water Pollution Control Technology, Research Center for Eco-Environmental Sciences, Chinese Academy of Sciences, Beijing 100085, China

c Center for Innovative Drug Research, Chongqing University, Chongqing 400044, China 
was likely to transfer its proton to amino zwitterionic molecule $\mathrm{A}^{ \pm}$to form an ion-pair complex in the $3.5<\mathrm{pH}<5.0$ range. It was also concluded that proton-transfer and cation-exchange reactions coexisted in the extraction of amino acid with D2EHPA. The complexation extraction was based on interaction between an electron pair donor and an electron pair acceptor or interaction between a Lewis base and a Lewis acid (the so-called complexing force). Due to the advantages of high capacity and high selectivity, reversible chemical complexation extraction has been the main method used to separate the polar, hydrophilic organic compounds in dilute solution by chelating extractant.22,26,27,31 Ihara et al. reported that six mixed-ligand nickel(II) and copper(II) chelates acted as receptors of amino acids in liquid-liquid extraction experiments between the 1,2-dichloroethane phase containing the metal chelates and the aqueous phase containing amino acids (L-phenylglycine, L-phenylalanine or L-tryptophan). The nickel(II) chelates effectively extracted amino acids from the aqueous phases under neutral conditions, forming octahedral ternary chelates. ${ }^{31}$ In the study of distribution behavior of L-tryptophan by extraction with D2EHPA, experimental data indicated the formation of both (1:1) and (1:2) L-tryptophan-D2EHPA (dimeric) complexes, and the complexes tended to cluster together, away from the low-polarity bulk solvent, but they did not cluster together when the polarity of the bulk solvent was improved. ${ }^{18}$ The achievements in mechanism exploration have made theoretical foundation for separation and purification of amino acids. However, the above four extraction mechanisms are mainly inferred by mathematical models or reaction equations, which are not conducive to promote the popularization and application of research ideas and achievements. Therefore, it is helpful to obtain the direct evidence by some appropriate experimental methods to further confirm the extraction mechanism of amino acids with typical extractants.

The essential amino acid L-Lys is an important amino acid applied as a special chemical in medicaments, chemical agents, food materials and feed additives. ${ }^{32-34}$ The separation and purification of L-Lys using an LLE method is prospective. ${ }^{7,35,36}$ Several authors have studied the separation and purification of L-Lys. Wu et al. studied the separation of low concentration L-Lys from aqueous solution by solvent sublation with dodecylbenzenesulforic acid (DBSA) as the surfactant and D2EHPA as the extractant. ${ }^{37}$ The results showed that the appropriate conditions of initial $\mathrm{pH}, \mathrm{D} 2 \mathrm{EHPA}$ concentration in the organic phase and DBSA concentration in the aqueous phase were $7.0,30 \%$ and $0.2 \mathrm{~g} \mathrm{~L}^{-1}$ respectively. The enrichment ratio and recovery ratio reached $13.26 \%$ and $79.57 \%$, respectively, at room temperature. In addition, Li et al. studied L-Lys extraction with the polyethylene glycol (PEG)/sodium sulfate aqueous two-phase system. ${ }^{38}$ The mass fraction of sodium sulfate and polyethylene glycol (PEG 1000) were controlled to be $14 \%$ and $20 \%$, respectively. The extraction efficiency and distribution coefficient of L-Lys standard liquid separately reached $96.5 \%$ and 0.033 at $30^{\circ} \mathrm{C}$ and $\mathrm{pH}=5.5$.

Nevertheless, few studies have directly investigated the extraction mechanism of L-Lys for better understanding of the extraction process and further increasing the extraction efficiency. Zhang et al. studied the transfer mechanism of L-Lys in liquid emulsion membrane (LEM). ${ }^{39}$ L-Lys transferred from feed solution to membrane phase when cations exchanged with carrier D2EHPA. Then free L-Lys diffused into the internal liquid membrane, followed by D2EHPA, and transferred into the internal phase when cation exchanged with strippant $\mathrm{H}^{+}$. In addition, Kaghazchi et al. reported the extraction of L-Lys from dilute aqueous solutions by emulsion liquid membrane. ${ }^{12}$ The forward extraction was based on the proton-exchange mechanism. However, the mechanism of L-Lys extraction by the method of LLE has never been reported.

A half-saturated fluorometric extraction experiment was performed to analyze L-Lys and CA-12-L-Lys extracted complex. The basic principle of the fluorescence method is described as follows. The amino groups of L-Lys react with o-phthalaldehyde (OPA) in the presence of $\beta$-mercapoethanol ( $\beta$-ME) to form fluorescent derivatives, which can be monitored at excitation and emission wavelengths of $340 \mathrm{~nm}$ and $455 \mathrm{~nm}$, respectively. L-Lys is a basic amino acid with $\alpha$-amino group and $\varepsilon$-amino group. ${ }^{40}$ Both amino groups can react with o-phthalaldehyde (OPA) to form the fluorescent derivatives L-Lys-OPA and L-Lys-(OPA) ${ }_{2}$ in weakly alkaline conditions. ${ }^{41}$ Theoretically, the $\alpha$-amino group and $\varepsilon$-amino group of L-Lys have the same reactivity with OPA, thus the half-saturated fluorescent derivative L-Lys-OPA can be obtained by making the molar ratio of OPA to L-Lys equal to 1:1. Furthermore, the fluorescent extracted complex can be formed via the reaction between a suitable anionic surfactant (as the extractant) and the free amino groups from L-Lys or L-Lys-OPA.

In this work, the common extractants sec-octylphenoxy acetic acid (CA-12), D2EHPA, trialkylamine (N235) and tributyl phosphate (TBP) were selected to extract and separate L-Lys. CA-12 is a novel carboxylic acid extractant ${ }^{42}$ with several advantages including easy preparation, stable composition, slow aqueous solubility, little emulsification during extraction and high extraction efficiency. ${ }^{43}$ CA-12 can ionize $\mathrm{H}^{+}$, and the carboxyl group of CA-12 and the amino group of L-Lys can form an ionic bond to achieve L-Lys extraction. ${ }^{44}$ Moreover, CA-12 had the best extraction efficiency $(62.8 \%)$ in preliminary experiments. D2EHPA is a cheap and stable phosphoric acid extractant, which can ionize a negatively charged ion. ${ }^{45}$ D2EHPA ${ }^{-}$and the amino group of L-Lys can form an ionic bond to achieve L-Lys extraction. The extraction efficiency of D2EHPA reached $49.7 \%$ in preliminary experiments. N235 (a mixture of tertiary amine $\mathrm{R}_{3} \mathrm{~N}$, where $\mathrm{R}=\mathrm{C} 8-\mathrm{C} 10$, molecular weight $=350-390$ ) is easily combined with protons and results in a quaternary ammonium cation. ${ }^{46}$ This cation reacts with the carboxyl group of L-Lys to form an ionic bond. The extraction efficiency of N235 was $11.9 \%$ in preliminary experiments. TBP is a neutral phosphorus-containing extractant having the advantages of stability at room temperature and long-term storage. ${ }^{47}$ Unfortunately, it was difficult to form stable ionic and hydrogen bonds with L-Lys so that the extraction efficiency of TBP was only $1.3 \%$ in preliminary experiments.

Sulfonated kerosene, also known as solvent naphtha \#260, was used as diluent, with the advantages of less aromatics, low toxicity, high safety, no odor, pure quality, low sulfur, environmental protection and strong solubility. ${ }^{48}$ Sulfonated kerosene was immiscible with L-Lys aqueous solution, and it showed high selectivity and stability in L-Lys extraction. ${ }^{7}$ Therefore, sulfonated kerosene was selected to be the diluent for extractant CA-12 in L-Lys extraction.

In this work, the mechanisms of L-Lys extraction with CA-12 in sulfonated kerosene were explored by atomic force microscopy (AFM), half-saturated fluorometric experiments and competition experiments. The local surface molecular structures of L-Lys, CA-12 and the extracted complex CA-12-L-Lys were directly observed by high-resolution AFM. In half-saturated fluorometric experiments, half of the amino groups in L-Lys were labeled by OPA to form the half-saturated fluorescent derivative L-Lys-OPA, which was then extracted by CA-12 in sulfonated kerosene; the fluorescence intensity before and after the extraction process was analyzed. Competition experiments between L-Lys and other amino acids, 


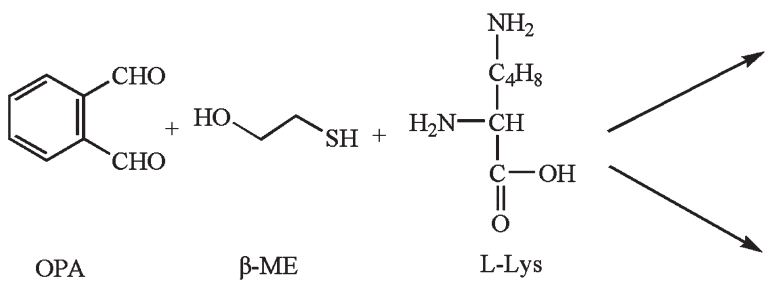

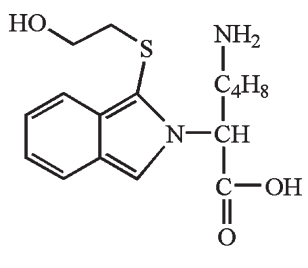

L-Lys - OPA<smiles>CCC(C(=O)O)n1cc2ccccc2c1SCCO</smiles>

L-Lys - (OPA)

Figure 1. Equations for the chemical reaction between L-Lys and OPA in the presence of $\beta$-ME in weak alkaline medium $(\mathrm{pH}=9.0)$ at room temperature.

namely L-arginine (L-Arg), L-aspartic acid (L-Asp) and L-alanine (L-Ala) were performed to further demonstrate the interaction between L-Lys and CA-12. In addition, the theoretical and experimental influences of $\mathrm{pH}$ on the extraction efficiency of L-Lys with CA-12 in sulfonated kerosene were compared and discussed. This work aims to provide solid strong experimental data to demonstrate that the chemical force of L-Lys extraction with CA-12 is an electrostatic interaction, namely the ionic bond between the amino group of L-Lys and the carboxyl group of CA-12.

\section{MATERIALS AND METHODS Reagents and chemicals}

Biochemical grade L-Lys, L-Arg, L-Asp and L-Ala were purchased from Shanghai Kangda Amino Acid Factory. Ninhydrin and 3, 5-dinitrosalicylic acid of analytical grade were provided by Eastern Sichuan Chemical Co. Ltd (Chongqing, China). Industrial grade N235, CA-12, D2EHPA and sulfonated kerosene were obtained from Laimei Yashi Chemical Co. Ltd (Shanghai, China). TBP of analytical-reagent grade was purchased from Chongqing Chemical Reagent Factory.

\section{Refining of extractants and sulfonated kerosene}

The extractants and sulfonated kerosene were refined by removing trace water-soluble impurities. CA-12, D2EHPA and N235 were insoluble in water, and the water content of industrial grade TBP was no more than $0.35 \%$. First, D2EHPA, TBP, N235, CA-12 and sulfonated kerosene were washed repeatedly with ultrapure water until the $\mathrm{pH}$ reached 7.0. Second, they were centrifuged for $10 \mathrm{~min}$ at $3000 \mathrm{rpm}$ and transferred to a separating funnel for one night. Finally, the aqueous phase was removed, while the purified extractants and sulfonated kerosene were collected and placed in brown mill mouth bottles.

\section{Preparation of extractive solutions}

Extractive solutions were prepared by slowly dissolving D2EHPA, TBP, N235 or CA-12 in deacidificated sulfonated kerosene under stirring to a desired concentration and placed in brown mill mouth bottles.

\section{Quantitative measurement of L-Lys}

L-Lys concentration was determined by a ninhydrin-colorimetry method in this work. L-Lys reacted with ninhydrin to form a blue-violet compound at $\mathrm{pH}=3.0$ and $100^{\circ} \mathrm{C}$. Then the compound was detected by $722 \mathrm{~S}$ visible spectrophotometer at $475 \mathrm{~nm}$. A perfect quadratic linear relationship between the concentration of L-Lys $\left(0-2.0 \mathrm{mg} \mathrm{mL}^{-1}\right)$ and the UV absorbance was achieved: $y=5.701 x^{2}+0.733 x+0.003\left(R^{2}=0.9997, S D=0.0043\right)$, where $y$ is the UV absorbance at $475 \mathrm{~nm}, x$ the concentration of L-Lys ( $\mathrm{mg} \mathrm{mL}^{-1}$ ), and $R$ the regression coefficient.

\section{Extraction experiments}

The extraction experiments were performed by making L-Lys solution (containing $0.5-1.5 \mathrm{~mol} \mathrm{~L}^{-1} \mathrm{~L}$-Lys) contact the extractive solutions in jacketed extractors for $15 \mathrm{~min}$ at $25^{\circ} \mathrm{C}$ under controlled $\mathrm{pH}$. The molar ratio of L-Lys solution to extractive solution was controlled to be 1:1. After reaching extraction equilibrium, the extracted liquids were centrifuged for $20 \mathrm{~min}$ at $10000 \mathrm{rpm}$ and then transferred to separating funnels to stand for $30 \mathrm{~min}$ to separate extraction phases and raffinate phases. The extraction phases were used to investigate the stripping extraction conditions, and the raffinate phases were used to measure the quantities of residual L-Lys. Then the extraction efficiencies $(E)$ and distribution coefficients $(D)$ of L-Lys were calculated using the following equations:

$$
\begin{gathered}
\text { extraction efficiency }(E, \%) \\
=\frac{\text { quantity of } L-\text { Lys in the extraction phase }}{\text { total quantity of } L-\text { Lys in initial } L-\text { Lys solution }} \times 100 \%
\end{gathered}
$$

$$
\begin{aligned}
& \text { distribution coefficient }(D) \\
& =\frac{\text { concentration of } L-\text { Lys in extraction phase }}{\text { concentration of } L-\text { Lys in raffinate phase }}
\end{aligned}
$$

In this study, batches of liquid-liquid extractions were performed to study appropriate conditions in which the variables evaluated were concentrations of L-Lys and extractants, phase ratio and extraction time. The sensitive ranges were 
determined by single factor experiments, and the appropriate extraction conditions were investigated by orthogonal experiments.

\section{AFM characterization}

A thin layer of dilute formaldehyde solution was pre-coated on two conductive microscopic glass slides and dried with sterile air. Then, approximately $0.05 \mathrm{~mL}$ dilute L-Lys solution (0.1 wt\%) and extracted liquid were dropped onto the two pre-coated microscopic glass slides and dried with sterile air again. Finally, AFM IPC-208B measurements of the above prepared samples were performed under the following conditions: tungsten probes (force constant $0.06 \mathrm{~N} \mathrm{~m}^{-1}$ ), scan range $10.5 \mathrm{~nm} \times 10.5 \mathrm{~nm}$, tapping mode imaging, scanning point by point at room temperature. The original image data were transmitted to a computer and further processed by G3DR software.

\section{Half-saturated fluorometric extraction experiments}

\section{(1) Principle of half-saturated fluorometric extraction}

L-Lys is a basic amino acid with $\alpha$-amino group and $\varepsilon$-amino group. Both free amino groups react with OPA to form fluorescent derivatives L-Lys-OPA and L-Lys-(OPA $)_{2}$ in the presence of $\beta$-mercaptoethanol $(\beta-\mathrm{ME})$ in weak alkaline medium $(\mathrm{pH}=9.0)$ at room temperature. The $\mathrm{pH}$ of mixtures was adjusted by adding $1 \mathrm{~mol} \mathrm{~L}^{-1} \mathrm{NaOH}$ to obtain the desired $\mathrm{pH}=9.0$. The chemical reaction equations are shown in Fig. 1.

The fluorescent derivative L-Lys-OPA can be monitored at excitation and emission wavelengths $340 \mathrm{~nm}$ and $455 \mathrm{~nm}$, respectively. The fluorescence intensity of L-Lys-OPA is positively correlated with L-Lys concentration over a certain range. As shown in Fig. 1, the stoichiometric ratio of OPA to L-Lys is 2:1 due to the same reactivity of $\alpha$-amino group and $\varepsilon$-amino group. When the molar ratio of OPA to L-Lys is lower than 1:1, OPA reacts with half of the amino group in L-Lys at most to form the half-saturated derivative L-Lys-OPA. Then the free amino group of L-Lys-OPA can react with the negatively charged extractant CA-12 to form fluorescent extracted complex. Consequently, the fluorescence intensity of the raffinate phase decreases with increasing concentration of L-Lys-OPA in the extraction phase. The extraction efficiency and the interaction between extractant and L-Lys-OPA can be analyzed by fluorescence spectrophotometry.

\section{(2) Quantitative measurement of half-saturated fluorometric extraction}

Based on this experiment, the half-saturated fluorometric standard curve was obtained. L-Lys solution with a concentration of $0.4 \mathrm{mg} \mathrm{mL}^{-1}$ was prepared using sodium bicarbonate buffer solution $(\mathrm{pH}=9.0)$ and $\beta$-ME $(300 \mu \mathrm{L})$. Then $5 \mathrm{~mL}$ of L-Lys solution was taken into each test tube containing $20 \mathrm{mg} \mathrm{mL}^{-1}$ OPA fluorescent reagent $(25-250 \mu \mathrm{L})$ and shaken up. Afterwards, the above test tubes were placed in a darkroom for $5 \mathrm{~min}$. Finally, the fluorescence intensity of the reaction solution in each test tube was monitored at excitation and emission wavelengths of $340 \mathrm{~nm}$ and $455 \mathrm{~nm}$, respectively. The half-saturated fluorometric standard curve was plotted with molar concentration of OPA as abscissa and fluorescence intensity (at $455 \mathrm{~nm}$ ) as vertical coordinate.

The half-saturated fluorometric extraction experiment for L-Lys was performed based on the molar ratio of OPA to L-Lys being 1:1. First, the quantified OPA solution and L-Lys solution were mixed in a test tube and shaken up. Second, the mixed solution was placed in a darkroom for $5 \mathrm{~min}$ to prepare the half-saturated fluorometric derivative L-Lys-OPA. Third, the quantified L-Lys-OPA solution and CA-12/sulfonated kerosene solution were mixed in a jacketed extractor and stirred for $20 \mathrm{~min}$. Afterwards, the extraction mixture was centrifuged for $20 \mathrm{~min}$ at $10000 \mathrm{rpm}$. Then, the centrifugate was transferred into a separating funnel to stand for $30 \mathrm{~min}$. Finally, the fluorescence intensities of the extraction phase and raffinate phase were measured. The extraction curves were plotted with molar concentration of OPA as abscissa and fluorescence intensities of extraction phase and raffinate phase as vertical coordinate, respectively.

\section{Competition experiment}

Several typical amino acids including basic L-Arg, acidic L-Asp and neutral L-Ala were selected as possible competitive reagents to L-Lys in the competition experiment. The molar ratios of L-Lys to each competitive amino acid (L-Arg, L-Asp and L-Ala) were controlled at $0.5,1.0,1.5,2.0$, and 2.5 , respectively. The extraction method of the competition experiment was in accordance with that detailed in the Extraction experiments section. The amino acids were analyzed by ninhydrin-colorimetry method. ${ }^{49}$ A curve reflecting the influences of the three typical amino acids on L-Lys extraction was plotted with the molar ratios of the other amino acids (L-Arg, L-Asp, L-Ala) to L-Lys as abscissa and the extraction efficiency as vertical coordinate, respectively.

\section{RESULTS AND DISCUSSION Verification of the electrostatic interaction between L-Lys and CA-12}

\section{AFM characterization}

AFM is a powerful characterization tool to reveal the local surface molecular structure and chemical composition of materials with superior spatial resolution, which has already been successfully applied to study the morphologies and interaction mechanisms of ion channels, ${ }^{50,51}$ protein extraction, ${ }^{52}$ short peptides ${ }^{6}$ and heat shock protein..$^{53}$ In this study, the high-resolution AFM was utilized to clearly reveal the molecular structure of L-Lys and CA-12, as well as the interaction between L-Lys and CA-12 in the extraction phase.

The AFM morphologies of L-Lys, CA-12 and the extracted complex CA-12-L-Lys are displayed in Figs 2, 3 and 4, respectively. The red and blue areas of AFM images with high probe density indicate the existence of atoms or groups of the observed samples, while no sample was found on the green areas. As shown in Figs 2 and 3 , the marked morphologies of L-Lys (Fig. 2(A)) and CA-12 (Fig. 3(A)) in AFM images are essentially consistent with their molecular models. Thus, visualization of the molecular structure of L-Lys and CA-12, as well as the structural relationship between L-Lys and CA-12 in the extraction phase, by AFM at atomic level is reliable.

Figure $4(A)$ shows the morphology of the extracted complex CA-12-L-Lys. There are massive chain-ring intermolecular structures, including the structure/morphology of two cyclic compounds approaching one chain compound (cyclic-chain-cyclic structure). As shown in Fig. 4(B), the chain-like molecule in the marked morphology of the so-called 'cyclic-chain-cyclic structure' is essentially consistent with that in Fig. 2(B) (namely L-Lys), while the cyclic-like molecule is consistent with that in Fig. 3(B) (namely CA-12). Moreover, it can be seen from Fig. 4(B) that both the two amino groups in the L-Lys molecule and the $\mathrm{O}$-atom of $\mathrm{C}-\mathrm{O}$ 
A

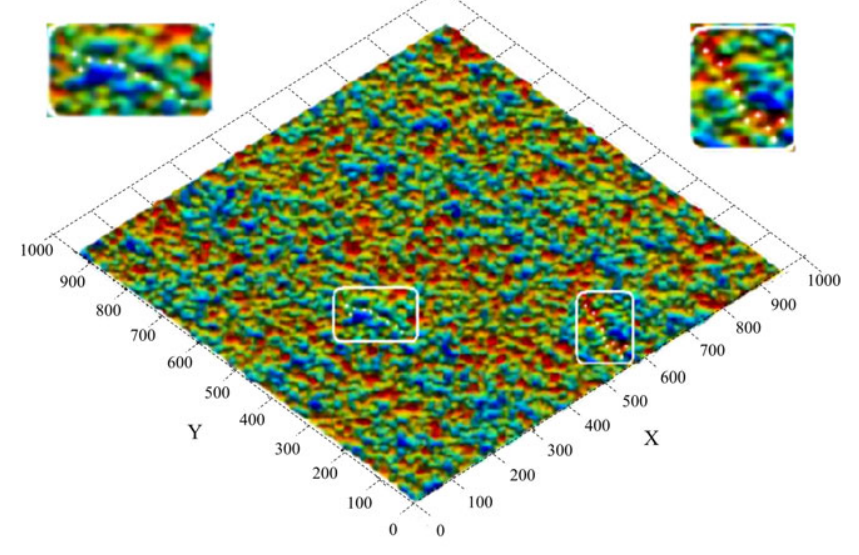

B

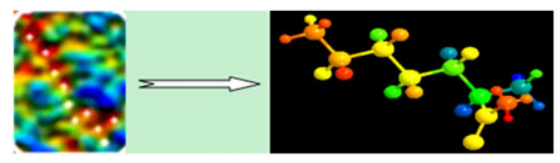

Figure 2. AFM image and molecular model of L-Lys (the full-length of $X$ and $Y$ directions are both $10.5 \mathrm{~nm}$ in the AFM image).

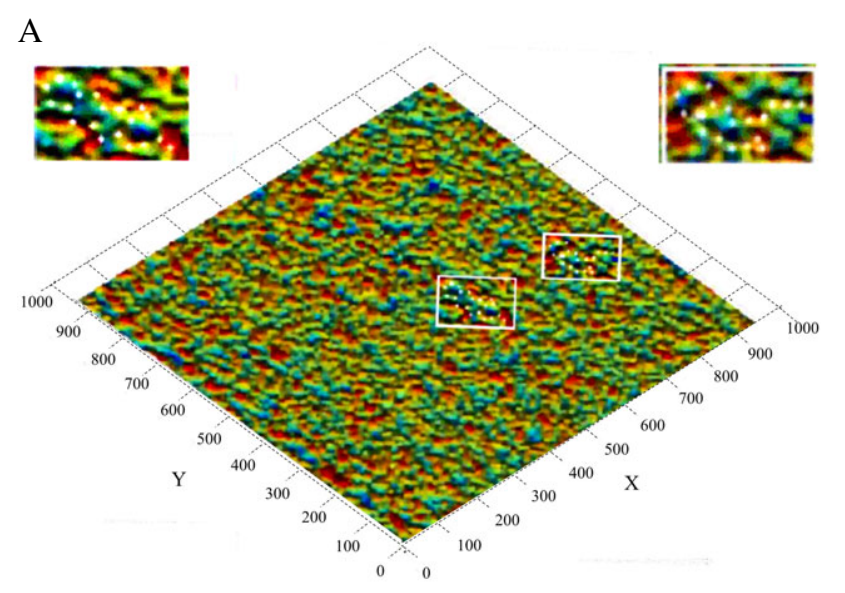

B

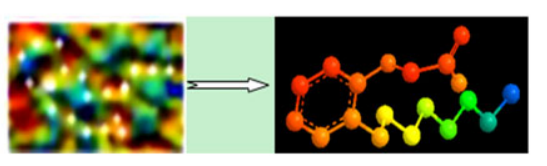

Figure 3. AFM image and molecular model of CA-12 (the full-length of $X$ and $Y$ directions are both $10.5 \mathrm{~nm}$ in the AFM image).

in the CA-12 molecule are arranged in a line and able to form an ionic bond. These observations mean that the mechanism of L-Lys extraction with CA-12 is based on the electrostatic attraction between the carboxyl group and the amino group. Therefore, it can be concluded that direct evidence of the electrostatic interaction between L-Lys and CA-12 in the extraction phase is successfully obtained by high-resolution AFM.

\section{Half-saturated fluorometric analysis}

As shown in Fig. 1, both amino groups of L-Lys can react with OPA to form the fluorescent derivatives L-Lys-OPA and L-Lys-(OPA) in the presence of $\beta$-ME in weak alkaline medium, where the stoichiometric ratio of OPA to L-Lys is 2:1. Thus, when the molar
A

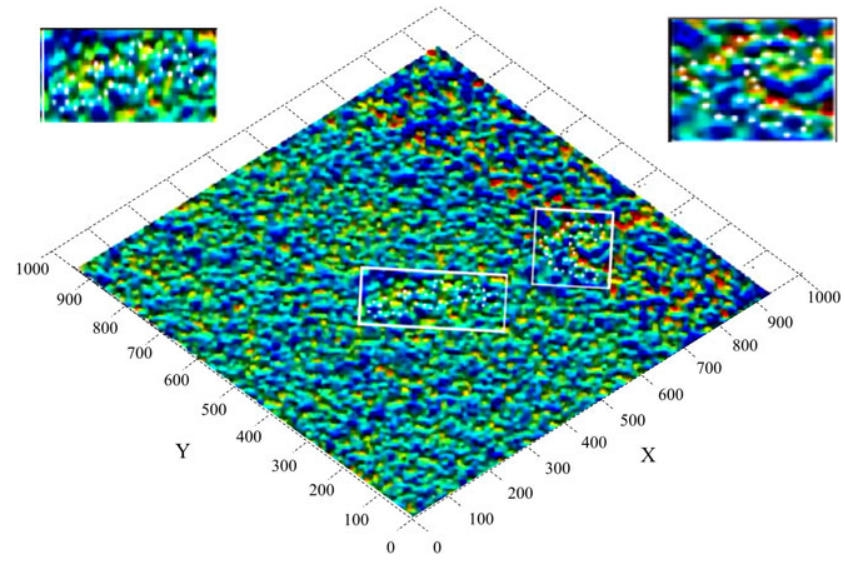

B

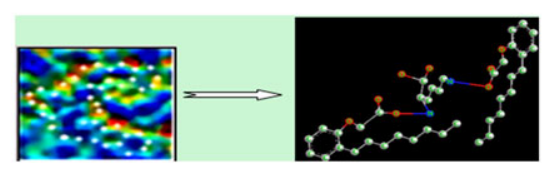

Figure 4. AFM image and molecular model of CA-12-L-Lys (the full-length of $X$ and $Y$ directions are both $10.5 \mathrm{~nm}$ in the AFM image).

ratio of OPA to L-Lys is lower than 1:1, all of the OPA molecules will react with L-Lys while less than half of the amino group can be consumed to form the half-saturated fluorescent derivative L-Lys-OPA. The free amino group of L-Lys-OPA can react with the negatively charged extractant CA-12 to form a kind of fluorescent extracted complex. Therefore, it is feasible to use the half-saturated fluorescent derivative L-Lys-OPA to explore the electrostatic interaction in the extraction process.

Figure 5 shows the relationship between OPA dosage and the fluorescence intensity of stock solution and extracted liquid, respectively. There was $30 \mu \mathrm{mol}$ L-Lys in the stock solution, namely $60 \mu \mathrm{mol}$ amino groups. Thus, when the OPA dosage was less than $60 \mu \mathrm{mol}$, the fluorescence intensity of the stock solution was positively correlated with OPA amount. If the OPA dosage was $60 \mu \mathrm{mol}$, the molar ratio of amino group to OPA was 1:1, which indicated that the generated derivative L-Lys-(OPA $)_{2}$ in the stock solution had the strongest fluorescence intensity. If the OPA dosage was more than $60 \mu \mathrm{mol}$, the fluorescence intensity of the stock solution was no longer changed. This result shows that the fluorescence intensity of the stock solution depends on the generated derivatives L-Lys-OPA and L-Lys-(OPA $)_{2}$. The fluorescence intensity of L-Lys-(OPA) ${ }_{2}$ is two times of that of L-Lys-OPA. It also reveals that all OPA molecules can participate in this reaction, with a reaction efficiency of $100 \%$.

In the extracted liquid, the fluorescence intensity first increased with increasing OPA amount until the OPA dosage was $30 \mu \mathrm{mol}$, and then decreased to the minimum value when the OPA dosage was $60 \mu \mathrm{mol}$. The fluorescence of extracted liquid came from the fluorescent extracted complex generated by the reaction between CA-12 and the half-saturated fluorescent L-Lys-OPA. In this extraction reaction, the carboxyl group of CA-12 reacts with the free amino group of the half-saturated fluorescent derivative L-Lys-OPA via electrostatic attraction. In other words, the fluorescence intensity of the extracted complex depends on the amount of L-Lys-OPA generated in the stock solution. As described above, when the OPA dosage was not more than $30 \mu \mathrm{mol}$, the generated amount of L-Lys-OPA increased with increasing dosage of OPA 


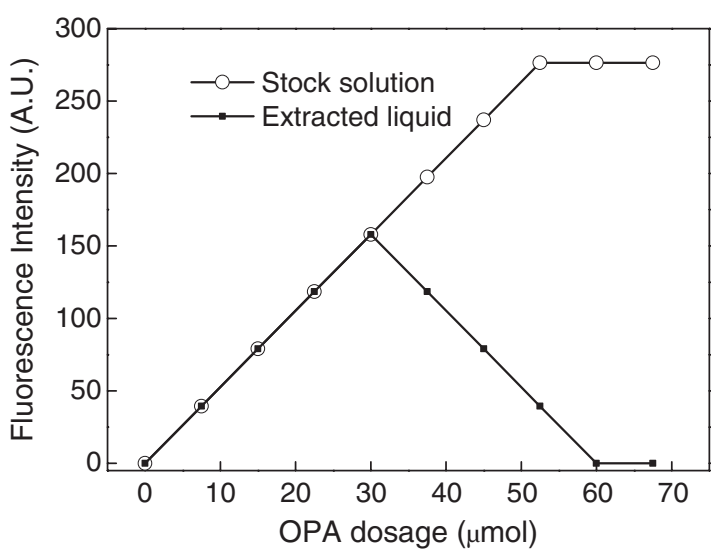

Figure 5. Fluorescence curve of L-Lys extraction with CA-12. The concentration and volume of L-Lys solution were $0.4 \mathrm{mg} \mathrm{mL}^{-1}$ and $10 \mathrm{~mL}$, respectively. The concentration of OPA reagent was $20 \mathrm{mg} \mathrm{mL}^{-1}$.

and reached a maximum at $30 \mu \mathrm{mol}$ OPA dosage. When the OPA dosage was more than $30 \mu \mathrm{mol}$, OPA reacted with L-Lys-OPA to form L-Lys-(OPA) 2 which could not react with extractant CA-12. As a result, the amount of L-Lys-OPA in the stock solution began to decrease and reached a minimum at $60 \mu$ mol OPA dosage. A similar tendency was observed in fluorescence intensity of the extracted liquid. This result also shows that the chemical force of L-Lys extraction with CA-12 is an electrostatic interaction between the carboxyl group and the amino group.

\section{Ion competition from other amino acids}

The influence of ion competition from other amino acids on L-Lys extraction with CA-12 in sulfonated kerosene is depicted in Fig. 6. It is obvious that the extraction efficiency of L-Lys is influenced to different degrees by the three typical amino acids. However, the influences of the three amino acids are similar, as the extraction efficiencies of L-Lys in the three curves all decrease with increasing molar ratios of the three amino acids to L-Lys. The three amino acids are all biological amino acids with $\alpha$-amino group and $\alpha$-carboxyl group, including the basic amino acid L-Arg, the neutral amino acid L-Ala and the acidic amino acid L-Asp. The different influences of the three amino acids on the extraction efficiency of L-Lys are mainly caused by their different side chain groups, since the contributions from their $\alpha$-amino groups and $\alpha$-carboxyl groups are similar.

The side chain group of L-Ala is methyl which does not react with the carboxyl group of CA-12, thus the influence of L-Ala on the extraction efficiency of L-Lys only reflects the effects of the $\alpha$-amino group and the $\alpha$-carboxyl group. The side chain group of L-Arg is guanidyl whose amino group has similar properties to the $\varepsilon$-amino group of L-Lys, resulting in competition between the two amino groups for the carboxyl group of CA-12. Consequently, the influence of L-Arg on the extraction efficiency of L-Lys contains the effects of not only the $\alpha$-amino group and $\alpha$-carboxyl group but also the guanidyl group. For the L-Asp, its side chain group $\beta$-carboxyl has the same charge as the carboxyl of CA-12, hence a mutual repulsion between the two carboxyl groups subtracts the influence of L-Asp on the extraction efficiency of L-Lys. Therefore, among the three typical amino acids, the basic amino acid L-Arg and the acidic amino acid L-Asp are determined to be the most and the least competitive amino acid, respectively. Based on the comparisons, the contribution of L-Arg to the extraction is

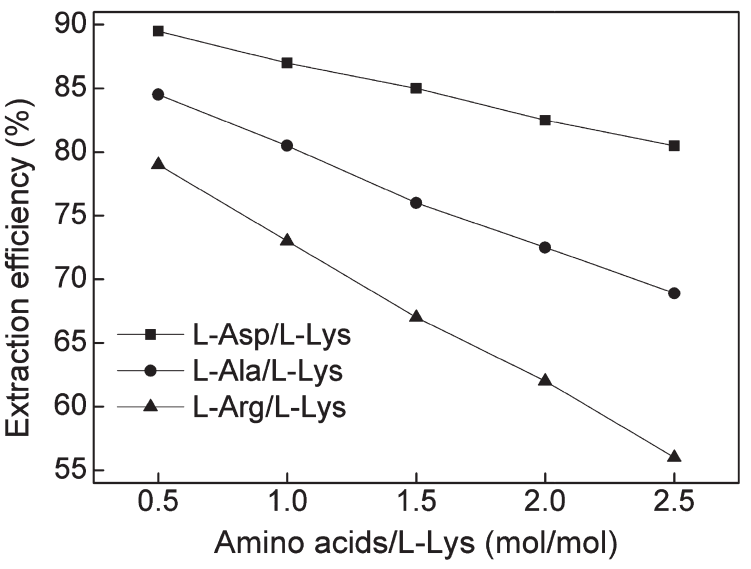

Figure 6. Influences of three typical amino acids on L-Lys extraction with CA-12 in sulfonated kerosene ( $\mathrm{L}-\mathrm{Lys}$ concentration $=0.4 \mathrm{mg} \mathrm{L}^{-1}, \mathrm{pH}$ of the initial solution $=10.0$, volume phase ratio $=1: 1$, extraction time $=15 \mathrm{~min}$ ).

Table 1. Extraction efficiencies of several extractants for L-Lys ${ }^{a}$

\begin{tabular}{|c|c|c|c|c|}
\hline Extractant & TBP & N235 & D2EHPA & CA-12 \\
\hline Extraction efficiency (\%) & 1.3 & 11.9 & 49.7 & 62.8 \\
\hline
\end{tabular}<smiles>O=C(O)COc1ccccc1-c1ccccc1</smiles>

CA-12<smiles>[R1]N([R1])C</smiles>

$\mathrm{N} 235$<smiles>CCCCCC(CC)COP(=O)(O)OCC(CC)CCCC</smiles>

D2EHPA

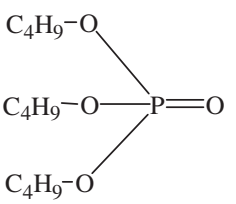

TBP
Figure 7. Structural formulas of the four extractants (N235 is a mixture of tertiary amine $\mathrm{R}_{3} \mathrm{~N}$, where $\mathrm{R}=\mathrm{C} 8-\mathrm{C} 10$, molecular weight $\left.=350-390\right)$.

equivalent to [L-Ala] and the positive effect of [guanidine- $\mathrm{NH}_{3}{ }^{+}$], while the contribution of L-Asp to the extraction is equivalent to [L-Ala] and the negative effect of $\left[\beta-\mathrm{COO}^{-}\right]$. This result is also consistent with the research conducted by Blaga et al. ${ }^{54}$

\section{Mechanisms of L-Lys extraction with several extractants in sulfonated kerosene}

It is widely known that the formation of a stable extracted complex is the key step in an extraction process. The chemical forces used to form the extracted complex include electrostatic interaction, hydrophobic interaction, hydrogen bond and coordination bond..$^{55}$ Moreover, higher extraction efficiency can be obtained by 


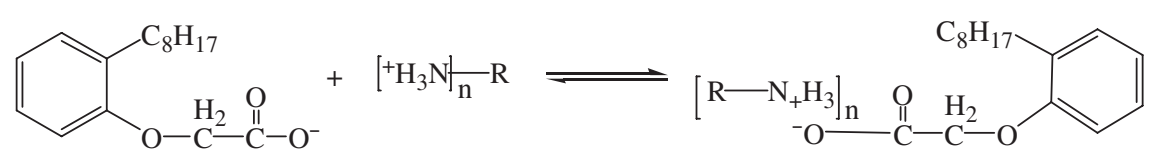

Figure 8. Reaction equation of L-Lys extraction with $\mathrm{CA}-12\left(\mathrm{H}_{3} \mathrm{~N}^{+}=\alpha\right.$-amino and $\varepsilon$-amino groups, $\mathrm{n}=1$ or 2$)$.

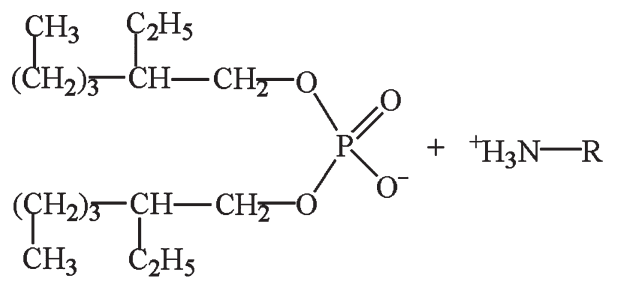<smiles>[R][NH2+][PH3+]P(=O)(OCC(CC)CC)OCC(CC)CC</smiles>

Figure 9. Reaction equation of L-Lys extraction with D2EHPA.

electrostatic interaction because the bond energy of ion-ion interaction is much higher than that of the other chemical bonds. This is also a reason why CA-12 is selected to extract L-Lys in sulfonated kerosene in this study. Table 1 lists extraction efficiencies of several extractants for L-Lys. Figure 7 displays the structural formulas of those extractants (CA-12, D2EHPA, N235 and TBP). Obviously, the CA-12/sulfonated kerosene system has the best extraction efficiency of $62.8 \%$ at $\mathrm{pH}=7.0$, which is increased by $26.4 \%$ and $427.7 \%$ compared with that of D2EHPA and N235, respectively.

TBP is a neutral phosphorus-containing extractant with an extraction functional group of phosphoryl $(\mathrm{P}=\mathrm{O})$ oxygen atom, hence it is difficult to form stable ionic and hydrogen bonds with the amino groups of L-Lys. As for the amine extractant N235, the electron density of its nitrogen atom will increase due to the electron denoting effects of its three R-substituents, making the nitrogen atom easily combined with a proton and resulting in a quaternary ammonium cation. ${ }^{56}$ This cation can combine with the carboxyl group of L-Lys to form an ionic bond in extraction process. However, quaternary ammonium compounds are stable only in acidic media, ${ }^{56}$ because the proton dissociation can be enhanced by the increasing $\mathrm{pH}$ and accordingly the extraction ability of quaternary ammonium cations decreased continuously. In neutral medium at $\mathrm{pH}=7.0$ in this study, there were only a few positive charges of N235, resulting in lower L-Lys extraction efficiency.

Both CA-12 and D2EHPA are acidic extractants, with the functional group of acetate carboxyl and oxyphosphate anion, respectively. Acetic acid is an organic weak acid, whose pKa is 4.76; while phosphate is a medium strong acid, whose pKa is 2.12. Theoretically, both CA-12 and D2EHPA can react with the $\alpha$-amino and $\varepsilon$-amino groups of L-Lys to form ionic bonds, as shown in Fig. 8 and Fig. 9, respectively. In this study, D2EHPA reacts with L-Lys to form a medium strong acid-weak base salt D2EHPA-O ${ }^{-+} \mathrm{H}_{2} \mathrm{~N}-\mathrm{Lys}$, while CA-12 reacts with L-Lys to form a weak acid-weak base salt CA-12-COO ${ }^{-+} \mathrm{H}_{2} \mathrm{~N}$-Lys which has a much lower ionization degree than D2EHPA-O ${ }^{-+} \mathrm{H}_{2} \mathrm{~N}$-Lys. As a result, the extraction efficiency of CA-12 is higher than that of D2EHPA, because the stability of CA-12-COO${ }^{-+} \mathrm{H}_{2} \mathrm{~N}$-Lys is significantly higher than that of D2EHPA-O ${ }^{-+} \mathrm{H}_{2} \mathrm{~N}$-Lys. ${ }^{57}$

As mentioned above, CA-12 is an organic weak acid, whose carboxyl dissociation is influenced by acidity; while L-Lys is a basic amino acid, and the charge properties of its amino groups are also influenced by acidity. Accordingly, in this study, the electrostatic interaction between CA-12 and L-Lys is controlled by acidity, so is the extraction efficiency. The $\mathrm{pK}_{\mathrm{a}}$ of CA-12 carboxyl and the
$\mathrm{pH}$ at the carboxyl's total dissociation are calculated to be 3.52 and 8.53 , respectively. ${ }^{58}$ Theoretically, when $\mathrm{pH}$ is less than 8.53 , the concentration of CA-12 anion is positively correlated with $\mathrm{pH}$, resulting in an increased extraction efficiency of L-Lys until reaching the maximum at $\mathrm{pH}=8.53$. The $\mathrm{pK}_{\mathrm{a}}$ values of $\alpha$-amino and $\varepsilon$-amino groups of L-Lys are calculated to be 8.95 and 10.53, respectively, hence the two amino groups are positively charged when $\mathrm{pH}$ is less than 7.0. However, the positively charged amino groups decrease gradually with increasing $\mathrm{pH}$ when $\mathrm{pH}$ is more than 7.0. Consequently, the concentration of CA-12-L-Lys and the extraction efficiency of L-Lys would be reduced. Therefore, the theoretical curve of the extraction efficiency versus the $\mathrm{pH}$ would be a polygonal line with $\mathrm{pH}=7.0$ as the vertex.

As shown in Fig. 10, with increasing $\mathrm{pH}$, the experimental extraction efficiency increased significantly first $(\mathrm{pH}<4.0$, curve slope of 7.91 ), then increased slowly ( $\mathrm{pH}=4.0$ to 8.0 , curve slope of 3.42 ), and later increased significantly again $(\mathrm{pH}>8.0$, curve slope of 13.95). This result is obviously different from that of the theoretical curve mentioned above, and the possible reasons are described as follows. (1) When $\mathrm{pH}$ is below 4.0, there are only a few dissociated CA-12 carboxyls due to its low $\mathrm{pK}_{\mathrm{a}}$ value (3.52); when the $\mathrm{pH}$ is raised, the dissociation of CA-12 carboxyl and the extracted complex can be increased significantly. (2) When $\mathrm{pH}$ exceeds 4.0, most of the CA-12 carboxyls are dissociated; although the $\mathrm{pH}$ is raised to 8.0 continually, the dissociation of CA-12 carboxyl and extracted complex increase slowly. (3) When $\mathrm{pH}$ is higher than 8.0, CA-12 carboxyl is completely dissociated, achieving the maximum extraction ability; however, much of the sulfonated kerosene sulfonic groups $\left(\mathrm{SO}_{3} \mathrm{H}^{-}\right)\left(\mathrm{pK}_{\mathrm{a}}=7.0\right)$ are dissociated, thus the extraction efficiency can be increased significantly again.

\section{CONCLUSIONS}

The conclusions drawn from this study of the extraction mechanisms of L-Lys with CA-12 in sulfonated kerosene are summarized below.

(1) The chemical force of L-Lys extraction with CA-12 is based on the electrostatic interaction between the carboxyl group and the amino group. Direct evidence of the electrostatic interaction between L-Lys and CA-12 in the extraction phase was successfully obtained by high-resolution AFM.

(2) It is feasible to use the half-saturated fluorometric extraction experiment to study the electrostatic interaction during the extraction process: CA-12 reacts with half-saturated derivative 


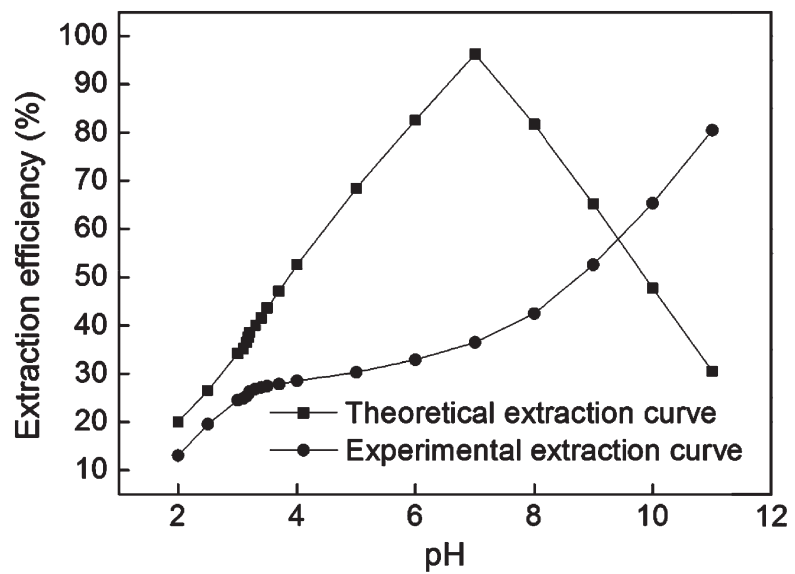

Figure 10. Influence of $\mathrm{pH}$ on the extraction efficiency of L-Lys with CA-12 in sulfonated kerosene (L-Lys concentration $=0.2 \mathrm{mg} \mathrm{mL}^{-1}$, volume phase ratio $=1: 1$, extraction time $=15 \mathrm{~min}$ ).

L-Lys-OPA to form the fluorescent extracted complex via electrostatic interaction.

(3) The basic amino acid L-Arg and the acidic amino acid L-Asp were determined to be the most and the least competitive amino acid, respectively; the influence of ion competition from L-Arg and L-Asp on the extraction efficiency of L-Lys could be described as $[\mathrm{L}-\mathrm{Ala}]+\left[\right.$ guanidine- $\left.\mathrm{NH}_{3}{ }^{+}\right]$and [L-Ala $]-\left[\beta-\mathrm{COO}^{-}\right]$, respectively.

(4) The analysis of $\mathrm{pH}$ influence on the extraction efficiency further indicates that the chemical force of L-Lys extraction with CA-12 in sulfonated kerosene is by electrostatic interaction.

In conclusion, the mechanisms of L-Lys extraction with CA-12 in sulfonated kerosene were comprehensively studied in this work. The chemical force between L-Lys and CA-12 was successfully demonstrated to be via an ionic bond based on the electrostatic interaction.

\section{ACKNOWLEDGEMENTS}

This research was supported by the National Natural Science Foundation of China (Grant 21106191), the State Key Laboratory of Materials-Oriented Chemical Engineering (Grant KL14-11), and Fundamental Research Funds for the Central Universities (project No. CDJXS12220003, CQDXWL-2013-019).

\section{REFERENCES}

1 Zhang PL, Luo JJ, Tang KW and Yi JM, Kinetics of enantioselective liquid-liquid extraction of phenylglycine enantiomers using a BINAP-copper complex as chiral selector. Chem Paper 68:1317-1324 (2014).

2 Tang KW, Zhang $\mathrm{H}$ and Zhang $\mathrm{PL}$, Continuous separation of alpha-Cyclohexyl-mandelic acid enantiomers by enantioselective liquid-liquid extraction in centrifugal contactor separators: experiments and modeling. Ind Eng Chem Res 52:3893-3902 (2013).

3 Lux S and Siebenhofer M, Investigation of liquid-liquid phase equilibria for reactive extraction of lactic acid with organophosphorus solvents. J Chem Technol Biotechnol 88:462-467 (2013).

4 Okubo Y, Maki T, Aoki N, Hong Khoo T, Ohmukai Y and Mae K, Liquid-liquid extraction for efficient synthesis and separation by utilizing micro spaces. Chem Eng Sci 63:4070-4077 (2008).

5 Zhou X, Su Y and Zhao S, Preparation of chitosan-quercetin resin microspheres and its adsorption properties for flavonoids. Sep Sci Technol 48:941-946 (2013).
6 Zhou X, Jin T, Dong L, Zheng S and Xiao J, Liquid-liquid extraction of GSH using DEHAP/octanol reverse micelles. Sep Sci Technol 44:3632-3649 (2009).

7 Zhang J, Zhou XH, Wang D, Zhou X, Yun F and Tan SY, A comprehensive study of extraction of L-Lysine with Sec-Octylphenoxy acetic acid in sulfonated kerosene. Sep Sci Technol 49:1349-1356 (2014).

8 Lin SH, Chen CN and Juang RS, Extraction equilibria and separation of phenylalanine and aspartic acid from water with di(2-ethylhexyl)phosphoric acid. J Chem Technol Biotechnol 81:406-412 (2006).

9 Ruiz MO, Escudero I and Horta Al, Extraction equilibria of $\alpha$-phenylglycine and aspartic acid with TOMAC-impregnated resins. Chem Eng Sci 62:2737-2749 (2007).

10 Hilton $M$ and Armstrong DW, Evaluation of a chiral crown etherlc column for the separation of racemic amines. J Liquid Chromatog 14:9-28 (1991).

11 Raizada P, Vyas V and Sharma U, Liquid membrane extraction and transport of amino acids using calix 6 arene. Indian J Chem Technol 17:267-273 (2010).

12 Kaghazchi T, Kargari A, Yegani R and Zare A, Emulsion liquid membrane pertraction of L-lysine from dilute aqueous solutions by D2EHPA mobile carrier. Desalination 190:161-171 (2006).

13 Hamdi A, Souane R, Kim L, Abidi R, Mutihac L and Vicens J, Extraction behaviour of amino acid esters by functionalised calix 4 arenes. J Incl Phenom Macrocycl Chem 64:95-100 (2009).

14 Liu YS and Dai YY, Distribution behavior of alpha-amino acids and aminobenzoic acid by extraction with trioctylamine. Sep Sci Technol 38:1217-1228 (2003).

15 Paull DH, Abraham CJ, Scerba MT, Alden-Danforth E and Lectka T, Bifunctional asymmetric catalysis: cooperative lewis acid/base systems. Accounts Chem Res 41:655-663 (2008)

16 Reyes-Rangel G, Bandala Y, Garcia-Flores F and Juaristi E, Asymmetric allylation of -ketoester-derived N-benzoylhydrazones promoted by chiral sulfoxides/N-oxides lewis bases: highly enantioselective synthesis of quaternary-substituted-allyl-amino acids. Chirality 25:529-540 (2013).

17 Li ZW, Wu WS, Du ZY and Hao XY, Structure and interaction between the BMIM Ala alanine anion and the 1-butyl-3-methylimidazolium cation in ion pairs. J Struct Chem 54:676-683 (2013).

18 Liu YS, Dai YY and Wang JD, Distribution behavior of L-tryptophane by extraction with di(2-ethylhexyl) phosphoric acid. Sep Sci Technol 35:1439-1454 (2000).

19 Pagba CV, Chi SH, Perry J and Barry BA, Proton-coupled electron transfer in tyrosine and a beta-hairpin maquette: reaction dynamics on the pico-second time scale. J Phys Chem B 119:2726-2736 (2015).

20 Xie YP, Jing KJ and Lu YH, Kinetics, equilibrium and thermodynamic studies of L-tryptophan adsorption using a cation exchange resin. Chem Eng J 171:1227-1233 (2011).

21 Moreira MJ and Ferreira LM, Kinetic studies for sorption of amino acids using a strong anion-exchange resin - effect of ionic strength. $J$ Chromatogr A 1092:101-106 (2005).

22 Grinstea Rr, Davis JC, Lynn S and Charles wRk, Extraction by phase separation with mixed ionic solvents - descriptive chemistry. Ind Eng Chem Prod Res Dev 8:218-227 (1969).

$23 \mathrm{Bi}$ PY, Dong HR, Yu HB and Chang L, A new technique for separation and purification of L-phenylalanine from fermentation liquid: flotation complexation extraction. Sep Purif Technol 63:487-491 (2008).

24 Liang B, Wu ZL, Hu B and Zheng HJ, Solvent sublation of L-arginine and its separation mechanism. Chin J Anal Chem 37:980-984 (2009).

25 King C, Handbook of Separation Process Technology. John Wiley, New York (1987).

26 Zhang TX, Jiang AP, Harrison JH and Chen SL, Pigment removal in anaerobically digested effluent through polyelectrolyte flocculation and liquid-liquid extraction. J Chem Technol Biotechnol 87:1098-1103 (2012).

27 Wu Z, Xu J, Sun Y, Xu N and Dai B, Clean synthesis of levulinic acid and its separation based on complexometric extraction. Mod Chem Ind 34:83-86 (2014)

28 Xun F, Junling L, Ying M, Li Z, Debao W and Zhengshui H, Amino acid extraction with AOT reverse micelle. Colloids and Surfaces $A$ : Physicochem Eng Aspects 179:1 - 10 (2001).

29 Fadnavis NW, Satyavathi B and Deshpande AA, Reverse micellar extraction of antibiotics from aqueous solutions. Biotechnol Prog 13:503-505 (1997). 
30 Yu P, Chang Z, Jin S, Wang S and Liu H, Progress and expectation of mechanisms research on liquid-liquid phase extraction and novel three-liquid-phase extraction. Chem Ind Eng Prog 9:006 (2009).

31 Ihara $\mathrm{Y}$, Kurose $\mathrm{S}$ and Koyama T, Extraction of unprotected amino acids by mixed-ligand nickel (II) and copper (II) chelates. Monatsh Chem 132:1433-1438 (2001).

32 Lopez-Arenas T, Anaya-Reza O, Quintero-Ramirez R and Sales-Cruz $M$, Modelling, simulation and dynamic analysis of the $L$-Lysine production process, in 24th European Symposium on Computer Aided Process Engineering, Pts a and B, ed by Klemes JJ, Varbanov PS and Liew PY, 1723-1728 (2014).

33 Ying HX, He X, Li Y, Chen KQ and Ouyang PK, Optimization of culture conditions for enhanced lysine production using engineered Escherichia coli. Appl Biochem Biotechnol 172:3835-3843 (2014).

$34 \mathrm{Xiao} \mathrm{Y}$ and $\mathrm{Zhou} \mathrm{XH}$, Synthesis and properties of a novel crosslinked chitosan resin modified by L-lysine. React Funct Polym 68:1281-1289 (2008).

35 Pineros I, Ballesteros P and Lastres JL, Extraction and LC determination of lysine clonixinate salt in water/oil microemulsions. J Pharm Biomed Anal 27:747-754 (2002).

36 Oshite S, Furukawa M and Igarashi S, Homogeneous liquid-liquid extraction method for the selective spectrofluorimetric determination of trace amounts of tryptophan. Analyst 126: 703-706 (2001).

37 Wu Z, Liang B, Hu B and Zheng H, Separation of L-Lysine by solvent sublation. Sep Purif Technol 66:237-241 (2009).

38 Li QY, Yuan FM and Wu JY, L-Lysine extraction with polyethylene glycol (PEG) / sodium sulfate aqueous two-phase system. Food Fermn Ind 40:221-226 (2014).

39 Zhang J, Yang XN, Tao TY and Gao B, Extraction behaviors of L-Lysine in liquid emulsion membrane. Chin Brew 31:137-139 (2012).

40 Author, Novel insulin derivative having side chain attached either to alpha-amino group of the $\mathrm{N}$-terminal amino acid residue or epsilonamino group of lysine of parent insulin $B$ chain through an amide bond, useful for treating diabetesWO2006082205-A1; EP1846447A1; US2008171695-A1; JP2008528659-W; US2010227796-A1; EP2292653-A1; US2012208749-A1; EP1846447-B1; JP5410020-B2; EP2292653-B1; ES2490243-T3; US8859493-B2.

41 Wu Y, Hu Y, Cai J, Ma S, Wang X and Chen Y, The Analysis of morphological distortion during AFM study of cells. Scanning 30:426-432 (2008).

42 Yang HL, Chen J, Wang W, Cui HM, Zhang DL and Liu Y, Extraction kinetics of lanthanum in chloride medium by bifunctional ionic liquid A336 CA-12 using a constant interfacial cell with laminar flow. Chinese J Chem Eng 22:1174-1177 (2014).

43 Tian M, Song N, Wang D, Quan X, Jia Q, Liao W et al., Applications of the binary mixture of sec-octylphenoxyacetic acid and 8-hydroxyquinoline to the extraction of rare earth elements. Hydrometallurgy 111:109-113 (2012).

44 Wang W, Yang H, Liu Y, Cui H and Chen J, The application of biodiesel and sec-octylphenoxyacetic acid (CA-12) for the yttrium separation. Hydrometallurgy 109:47-53 (2011).
45 Lin SH and Chen CN, Simultaneous reactive extraction separation of amino acids from water with D2EHPA in hollow fiber contactors. $J$ Membr Sci 280:771-780 (2006).

46 Fang DW, Shan WJ, Yan Q, Li D, Xia LX and Zang SL, Extraction of rhenium from sulphuric acid solution with used amine N235. Fluid Phase Equil 383:1 -4 (2014).

47 Das D, Juvekar VA and Bhattacharya R, Problem associated with the use of TBP for LEM extraction of $\mathrm{U}(\mathrm{VI})$ and attempt to overcome this problem using other ligands.J Radioanal Nucl Chem 304:1027-1042 (2015).

48 Zhang $X Y$, Lou XD, Yin GY and Zhang YJ, Extraction and separation of $\mathrm{Ga}$ (III) and $\mathrm{TI}(\mathrm{I})$ or $\mathrm{In}(\mathrm{III})$ and $\mathrm{TI}(\mathrm{I})$ from chloride media with CA-12 in sulphonating kerosene. Rare Metals 23:6-9 (2004).

49 Bali A and Gaur P, A novel method for spectrophotometric determination of pregabalin in pure form and in capsules. Chem Cent J 5:59 (2011).

50 Yang R, Xi N, Lai KWC, Patterson KC, Chen H, Song B et al., Cellular biophysical dynamics and ion channel activities detected by AFM-based nanorobotic manipulator in insulinoma beta-cells. Nanomed - Nanotechnol Biol Med 9:636-645 (2013).

51 Arce FMT, Jang $H$, Nussinov R and Lal R, Real-time Afm imaging of surface-induced oligomerization of the non-amyloidogenic $\mathrm{P} 3$ peptide: implications for membrane insertion and ion channel formation. Biophy J 98:110A-110A (2010).

52 Zhou XH and Weng YJ, Factors affecting trypsin extraction by AOT reversed micelles and observation by STM. Chinese J Chem Eng 14:407-412 (2006).

53 Pillet $F$, Lemonier S, Schiavone M, Formosa C, Martin-Yken H, Francois $\mathrm{JM}$ et al., Uncovering by atomic force microscopy of an original circular structure at the yeast cell surface in response to heat shock. BMC Biology 12:6 (2014).

54 Blaga AC, Galaction Al and Cascaval D, Separation of amino acids from their mixture by facilitated pertraction with D2EHPA. Chem Biochem Eng Q 22:439-446 (2008).

55 Wilson AM, Bailey PJ, Tasker PA, Turkington JR, Grant RA and Love JB, Solvent extraction: the coordination chemistry behind extractive metallurgy. Chem Soc Rev 43:123-134 (2014).

56 Ding M, Li J, Fu X, Zhou J, Tan H, Gu Q et al., Synthesis, degradation, and cytotoxicity of multiblock poly(epsilon-caprolactone urethane)s containing gemini quaternary ammonium cationic groups. Biomacromolecules 10:2857-2865 (2009).

57 Ferreira Q, Gomes PJ, Ribeiro PA, Jones NC, Hoffmann SV, Mason $\mathrm{NJ}$ et al., Determination of degree of ionization of poly(allylamine hydrochloride) (PAH) and poly 1- 4-(3-carboxy-4 hydroxyphenylazo) benzene sulfonamido -1,2-ethanediyl, sodium salt (PAZO) in layer-by-layer films using vacuum photoabsorption spectroscopy. Langmuir 29:448-455 (2013).

58 Li H, Liu J, Zhu L, Gao X, Wei S, Gu L et al., Recovery of Indium(III) from a hydrochloric acid medium with two types of solvent impregnated resins containing sec-octylphenoxy acetic acid. Solvent Extr Res Dev - Jpn 21:147-161 (2014). 